\title{
因HAD
}

ISSN-L: 2530-5115

(1) (1)(2)

DOI: http://doi.org/10.22585/hospdomic.v4i1.95

\section{Tendencias temporales de los patrones de búsqueda de información sobre servicio de asistencia sanitaria domiciliaria en España}

\section{Temporary trends in Information search patterns about home health care service in Spain}

\footnotetext{
María Sanz-Lorente

1. a. Universidad Miguel Hernández. Departamento de Salud Pública e Historia de la Ciencia, Campus de Sant Joan d'Alacant. Alicante. España.

1. b. Consellería de Sanidad Universal y Salud Pública de la Generalitat Valenciana. Centro de Salud Pública. Manises. España.
}

Correspondencia/Correspondence

María Sanz-Lorente

msanzlor@gmail.com

Recibido/Received

22.12.2019

Aceptado/Accepted

10.01.2020
Conflicto de Intereses/Competing interest La autora del trabajo declaran no tener ningún conflicto de interés 


\section{RESUMEN}

Objetivo: Analizar las tendencias temporales de los patrones de búsqueda de información sobre el servicio de asistencia sanitaria domiciliaria en España, a través de Google, desde el año 2004 a la actualidad.

Método: Estudio descriptivo transversal. Los datos se obtuvieron de la consulta directa a la herramienta «Google Trends» para España. Tema de búsqueda «servicio de asistencia sanitaria domiciliaria». Fecha de consulta 12 nov 2019.

Resultados: La tendencia de búsqueda obtenida presentó un gráfico con progresión ligeramente decreciente y ajuste a un modelo exponencial $\left(R^{2}=0,06 ; p=0,001\right)$. Al separar por época se obtuvo, en la 1. ${ }^{a}$ época un modelo lineal decreciente $\left(R^{2}=0,42 ; p<0,001\right)$ y posteriormente en la 2. ${ }^{a}$ época un modelo exponencial creciente $\left(R^{2}=0,57 ; p<0,001\right)$, existiendo diferencias entre la 1. ${ }^{\mathrm{a}}$ y la 2 . $^{\mathrm{a}}$ época a estudio: $38,57$ versus 29,60 ( $t=3,86 ; p<0,001)$.

Conclusión: La evolución del VBR que se obtuvo probablemente se deba a un cambio de preocupación social y esté ligado al vaivén de la información sobre los servicios de asistencia sanitaria domiciliaria en España. Futuras investigaciones deberían corroborar estas conclusiones ya que no dejan de ser una hipótesis que el actual estudio no está en condiciones de despejar. Además, estos nuevos estudios pueden confirmar, o no, la utilidad y limitaciones de "Google Trends» en el seguimiento de la necesidad de información sobre salud de la población.

Palabras clave: Servicios de Atención de Salud a Domicilio; Atención Domiciliaria de Salud; Acceso a la Información; Gestión de la Información; Alfabetización Informacional; Medios de Comunicación Sociales.

\section{ABSTRACT}

Objective: To analyze the temporary trends of the patterns of search for information about the home health care service in Spain through Google from 2004 to the present day.

Method: Cross-sectional descriptive study. The data were obtained from the direct consultation of the "Google Trends" tool for Spain. Search topic 'home health care service'. Date of consultation 12 jan 2019.

Results: The search trend obtained presented a graph with slightly decreasing progression and adjustment to an exponential model $\left(R^{2}=\right.$ 0.06 ; $p<0.001)$. Separating by period it was obtained, at the 1st epoch, a decreasing linear model $\left(R^{2}=0.42 ; p<0.001\right)$ and later in the $2 n d$ epoch a growing exponential model $\left(R^{2}=0.57\right.$; $p<0.001)$, there are differences between the 1st and the 2nd epoch / period to study: 38.57 versus $29.60(t=3.86 ; p<0,001)$.

Conclusion: The evolution of the RSV that was obtained is likely due to a change of social concern and is linked to the swings of information on home health care services in Spain. Future research should corroborate these findings as it is still a hypothesis that the current study is not in a position to clear. In addition, these new studies may or may not confirm the usefulness and limitations of Google Trends in monitoring the need for information about population health.

Keywords: Home Care Services; Home Nursing; Access to Information; Information Management; Information Literacy; Social Media. 


\section{INTRODUCCIÓN}

Dentro del ámbito sanitario, la evolución de la Web 2.0 también se ha hecho patente, y con el paso de los años se observa un cambio hacia una sociedad cada vez más informada sobre salud en Internet, hasta el punto de adoptar nuevos términos para nuestro vocabulario como el de eSalud. La llegada de las redes sociales (RRSS) digitales, consideradas el principal estandarte de este nuevo entorno digital, supuso una nueva vuelta de tuerca en la evolución de la comunicación hipermedia y en los modelos de comunicación tradicionales (1).

Actualmente, en un escenario marcado por el crecimiento en el uso de las RRSS y los recursos de interactividad, su inclusión en espacios digitales de comunicación en salud deviene un tema relevante. Durante la última década, el uso de datos basados en la Web sobre temas de salud pública, es decir, la «infodemiología», ha demostrado ser útil para evaluar diversos aspectos del comportamiento humano (2). En este sentido, la Web 2.0 ofrece oportunidades sin precedentes a la hora de buscar información sobre salud. De hecho, hace ya tiempo que se busca allí esta información, incluso antes que consultar con los profesionales (3).

Eysenbach (4), en el año 2009, acuñó el término de «infodemiología» o «infoepidemiología» como un conjunto emergente de métodos de información de salud pública para analizar el comportamiento de la búsqueda, la comunicación y la publicación en Internet. Es decir, la «infodemiología» observa y analiza el comportamiento basado en la Web para conocer la conducta humana real a fin de predecir, evaluar, e incluso prevenir problemas relacionados con la salud que surgen constantemente en la vida cotidiana (5).

La idea de que las poblaciones proporcionen datos sobre sus gustos e incluso sobre su enfermedad, a través del comportamiento de búsqueda de información en la Web, ya ha sido explorada en los últimos años (6). Johnson y Mehta (7), en el año 2014, ya demostraron, estudiando las tendencias de los motores de búsqueda, que eran herramientas válidas para integrarlas en la vigilancia en tiempo real de las infecciones de transmisión sexual. Otros autores utilizaron las tendencias de búsqueda en Google para comprobar la relación con los datos de enfermedad, por ejemplo en el campo de la influenza (8) o sobre el virus de la inmunodeficiencia humana (9).

Asimismo, en un anterior trabajo sobre las tendencias temporales de los patrones de búsqueda de información sobre cuidado domiciliario, se concluía la existencia de un creciente volumen de búsqueda relativo con relación al término "Cuidado Domiciliario", aunque se consideraba que serían necesarios un mayor número de estudios para conocer la utilidad y limitaciones epidemiológica de las investigaciones que usen los resultados de la búsqueda de información online (10).

Así pues, está más que demostrado que Google es un motor de búsqueda que facilita información -incluso sobre salud- a cualquier persona, que a través de los resultados obtenidos puede acceder fácilmente a los documentos existentes en la Red. Y, aunque no es el útil más conocido de Google, «Google Trends» es una herramienta, de acceso libre, que informa del volumen total de búsquedas realizadas por los usuarios de todo el mundo para exponer qué tan frecuentemente se busca un término y en qué lugares. Se puede afirmar que esta aplicación facilita la investigación sobre las ciencias de la salud en la era de Big Data. Los datos de búsqueda en Internet pueden proporcionar información valiosa sobre los patrones de enfermedad y el comportamiento de la población $(10,11)$.

En este contexto, este estudio tuvo como objetivo analizar las tendencias temporales de los patrones de búsqueda de información sobre el servicio de asistencia sanitaria domiciliaria en España, a través de Google, desde el año 2004 a la actualidad. 


\section{MATERIAL Y MÉTODO}

\section{Diseño}

Estudio descriptivo transversal.

\section{Fuente de obtención de la información}

Los datos se obtuvieron de la consulta directa, mediante acceso online, a Google Trends (https:// trends.google.es/).

\section{Herramienta}

Google Trends proporciona estadísticas normalizadas de las tendencias de Google para diferentes búsquedas desde el 1 de enero de 2004. Analiza las consultas para determinar cuántas pesquisas se realizaron con un determinado "Tema" o "Término de búsqueda", en comparación a la cantidad total de búsquedas realizadas en Google, excluyendo los volúmenes de búsqueda muy bajos o búsquedas duplicadas realizadas por el mismo usuario en un período de tiempo muy corto.

\section{Tema de búsqueda}

Se utilizó "servicio de asistencia sanitaria domiciliaria» como "Tema" y tomando los resultados, para España, de todas las categorías y de la categoría de salud. Hay que tener en cuenta que en esta herramienta la consulta utilizando un "Término" de búsqueda muestra las coincidencias de todos los términos de la consulta en el idioma especificado (Ejemplo: si se busca "queso" se obtendrán también resultados sobre "queso manchego", "queso tierno", etc.). En cambio, si se utiliza el vocablo como "Tema" se obtendrán los resultados de un de términos que comparten el mismo concepto en cualquier idioma. (Ejemplo: si se busca "Londres", la búsqueda incluye resultados de temas como los siguientes "capital del Reino Unido", "London" o "London city", entre otros). La fecha de consulta fue el 12 de noviembre de 2019.

\section{Obtención y almacenamiento de los datos}

Los resultados obtenidos fueron descargados en formato normalizado comma-separated values (CSV) que permitió su almacenamiento posterior en un archivo Excel. El control de la calidad de esta información se efectuó mediante dobles tablas, corrigiendo las posibles inconsistencias mediante la consulta con la tabla original descargada. Para el análisis estadístico se utilizó el programa Statistical Package for the Social Sciences (SPSS) para Windows, versión 22.0.

\section{Variables a estudio}

Volumen de búsqueda relativo (VBR): Resultado ofrecido por Google Trends cuyos valores se normalizan en una escala de 0 (un volumen de búsqueda relativo inferior al $1 \%$ del volumen máximo) a 100 (el volumen de búsqueda relativo alcanza su máximo). Por ejemplo, un VBR $=50$, representa el $50 \%$ de la proporción de búsqueda observada más alta durante el período a estudio. 
Para conocer la evolución del VBR se segmentó el período de estudio en dos épocas, tomando como punto de corte la mediana de la distribución de la edad de la búsqueda: 1. a época: de 2004 a 2011 y 2. época: de 2012 a 2019.

Evolución temporal: progreso de la tendencia de búsqueda a lo largo del tiempo.

\section{Análisis de los datos}

Para las variables cualitativas (Temas relacionados) se calcularon las frecuencias absoluta y relativa (porcentaje) y las variables cuantitativas (VBR y evolución temporal) mediante la media y su desviación estándar, la mediana, la amplitud intercuartílica (AIQ), el máximo y mínimo. Se analizó la existencia de asociación entre las variables cuantitativas para comprobar la significación de la diferencia de medias para muestras independientes, utilizando el test de la t de Student. Para obtener la relación entre variables cuantitativas se usó el coeficiente de correlación de Pearson. La evolución temporal de las tendencias de búsqueda se examinó mediante el análisis de regresión, calculándose el coeficiente de determinación. El nivel de significación utilizado en todos los contrastes de hipótesis fue $a \leq 0,05$.

\section{RESULTADOS}

A partir de la consulta realizada se obtuvo el VBR para el tema a estudio, en todas las categorías y de la categoría de salud, ver figura 1. EI VBR para cada una de las comunidades autónomas españolas puede consultarse en la figura 2.

Figura 1. Evolución anual del volumen de búsqueda relativo (VBR), para España, obtenido de Google Trends mediante el Tema "servicio de asistencia sanitaria domiciliaria», desde el 1 de enero de 2004 a 31 diciembre 2019

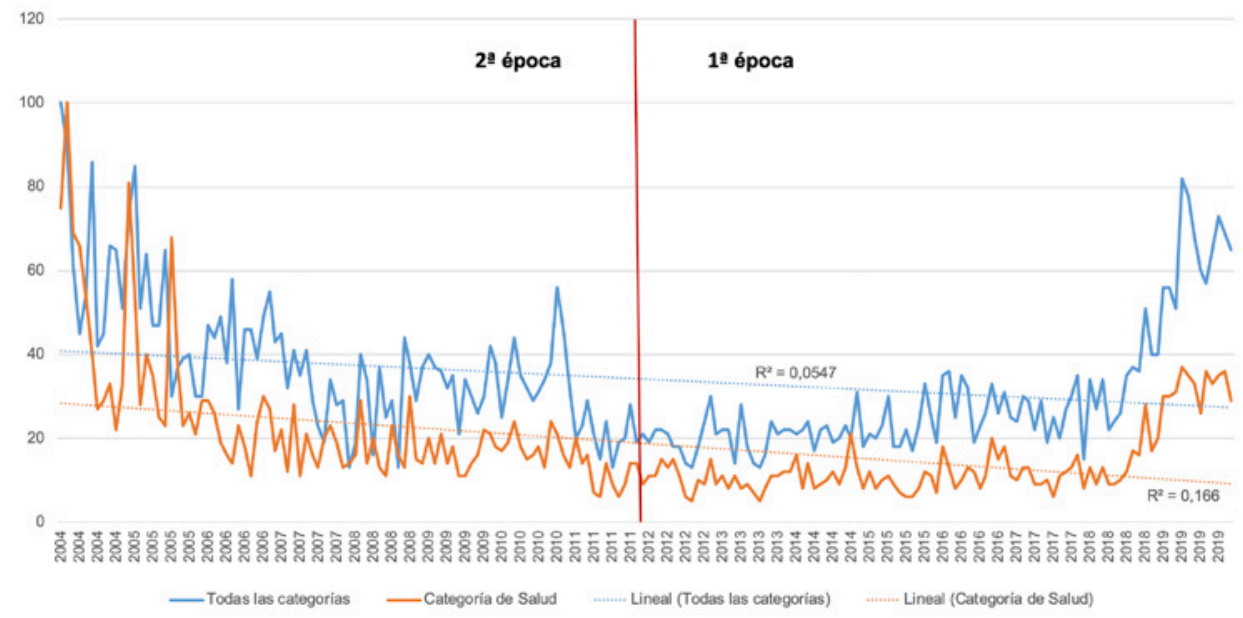


Figura 2.Volumen de búsqueda relativo (VBR), para el Tema «servicio de asistencia sanitaria domiciliaria», desde el 1 de enero de 2004 a 31 diciembre 2019, según comunidad autónoma española

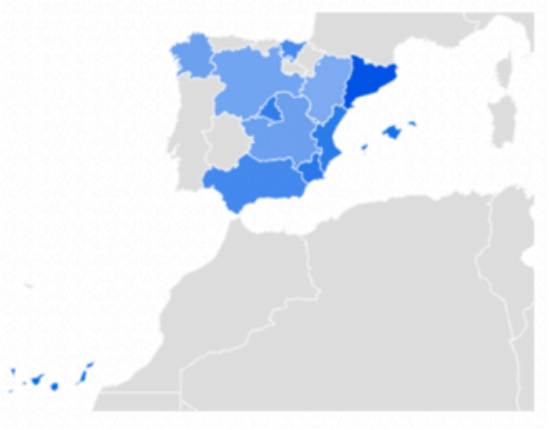

\begin{tabular}{|lcr|}
\hline Comunidad & $\begin{array}{c}\text { Todas las } \\
\text { categoría }\end{array}$ & $\begin{array}{c}\text { Categoría de } \\
\text { Salud }\end{array}$ \\
\hline Cataluña & 100 & 93 \\
\hline Canarias & 79 & 100 \\
\hline Islas Baleares & 75 & 84 \\
\hline Región de Murcia & 68 & 53 \\
\hline Comunidad Valenciana & 67 & 79 \\
\hline Comunidad de Madrid & 64 & 56 \\
\hline Andalucía & 55 & 58 \\
\hline País Vasco & 54 & 49 \\
\hline Castilla la Mancha & 36 & 48 \\
\hline Galicia & 35 & 34 \\
\hline Castilla León & 32 & 36 \\
\hline Aragón & 27 & 32 \\
\hline •El resto de las Comunidades Autónomas no presentaron resultados de VBR. \\
\hline
\end{tabular}

\section{Volumen de búsqueda relativo (VBR)}

Los estadísticos, para todo el periodo analizado del VBR para «servicio de asistencia sanitaria domiciliaria» y para todas las categorías, fueron: media 34,09 \pm 1,20; mediana 30; AIQ 19; máximo 100 y mínimo 13. Para la categoría de salud se obtuvo: media 18,81 $\pm 1,00$; mediana 14; AIQ 12; máximo 100 y mínimo 5.

El cociente medio, del conjunto del periodo analizado, fue de $2,07 \pm 0,05$, existiendo diferencias estadísticamente significativas entre los VBR de todas las categorías y los de la categoría de salud (34,09 versus 18,$81 ; t=9,77 ; p<0,001)$.

La correlación existente entre los VBR de todas las categorías y el VBR para la categoría de salud dio una correlación significativa, 1,00 a 0,76, en el nivel 0,01.

\section{Evolución temporal del VBR}

La tendencia de búsqueda obtenidas para todas las categorías presentó un gráfico con progresión ligeramente decreciente y ajuste a un modelo exponencial $\left(R^{2}=0,06 ; p=0,001\right)$. En el caso de la categoría de salud la evolución fue decreciente con ajuste igualmente a un modelo exponencial $\left(R^{2}=0,18 ; p<0,001\right)$ (figura 1).

Al separar por época se obtuvo, para todas las categorías, en la $1 .{ }^{a}$ época un modelo lineal decreciente $\left(R^{2}=0,42 ; p<0,001\right)$ y posteriormente en la 2 . $^{a}$ época un modelo exponencial creciente $\left(R^{2}=0,57 ; p<0,001\right)$. Además, se hallaron diferencias en la media del VBR entre la $1 .{ }^{a}$ y la $2 .{ }^{a}$ época a estudio: 38,57 versus 29,60 ( $t=3,86 ; p<0,001)$, ver figura 3 .

Para la categoría de salud, en la $1 .{ }^{a}$ época un modelo exponencial decreciente $\left(R^{2}=0,51 ; p<\right.$ $0,001)$ y posteriormente en la 2 . $^{a}$ época un modelo lineal creciente $\left(R^{2}=0,37 ; p<0,001\right)$ (figura 1). También se hallaron diferencias entre las medias según época: 23,65 versus 13,98 ( $t=5,16 ; p<$ $0,001)$, ver figura 3. 
Figura 3.Comparación de las medias del volumen de búsqueda relativo (VBR), para España, obtenido de Google Trends mediante el Tema «servicio de asistencia sanitaria domiciliaria», para todas las categorías y para la categoría de salud según época a estudio (1ª época: 2004 a 2011; $2^{a}$ época de 2012 a 2019)
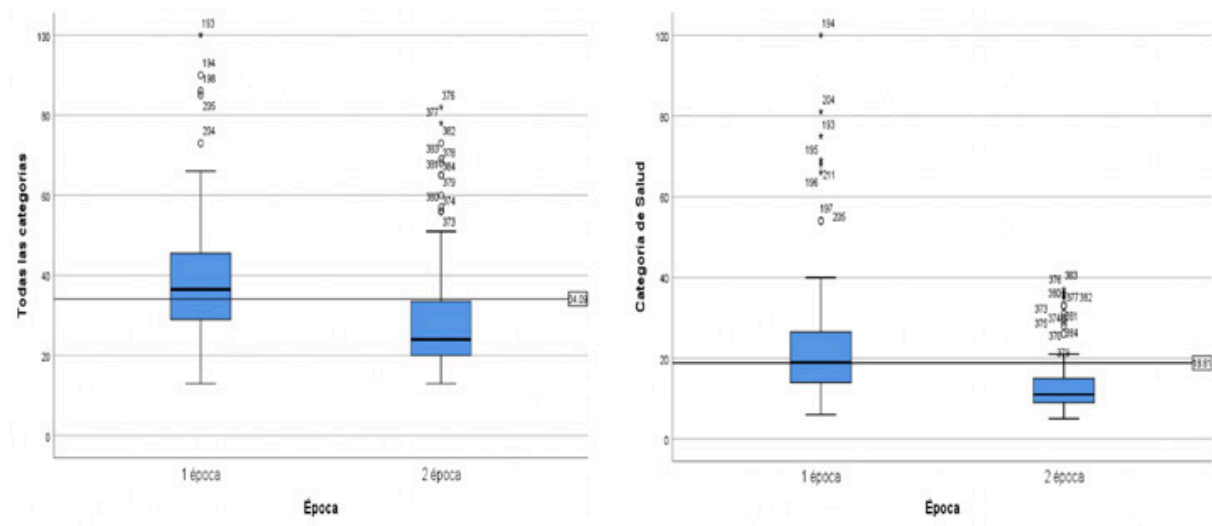

\section{DISCUSIÓN}

Los datos del VBR obtenidos con «Google Trends» permitieron conocer el interés del público en el tema a estudio. Como se ha comprobado, y en línea con un anterior trabajo (2), las tendencias de los motores de búsqueda son una herramienta que puede integrar la vigilancia, en tiempo real, de las necesidades de información sobre salud de la población.

Sin embargo, hay que tener en cuenta que se trata de un análisis de datos ecológicos y los resultados obtenidos pueden no coincidir con las búsquedas realizadas a nivel individual (12). Es decir, las tendencias son a nivel de población y no se debe concluir que solo las personas con interés en los servicios de asistencia sanitaria domiciliaria son aquellos que generan todo el volumen de búsqueda. También, existe incertidumbre acerca de la causa de las tendencias en las búsquedas (por ejemplo: aumento del interés por el tema seleccionado, aparición de noticias o atención de los medios de comunicación, etc.).

Asimismo, es complicado conocer la relación de los resultados con las diferencias en el acceso a Internet por región (2). Así, el interés observado en el VBR en Cataluña es posible que se deba a una buena implantación de las unidades de hospitalización a domicilio y su reconocimiento por las autoridades sanitarias de esa comunidad (13). Pero, la Comunidad Valenciana también presenta una buena implantación de los servicios de atención domiciliaria (14) y sus datos del VBR no se encuentran en los primeros lugares. Quizá, en el tema estudiado hayan tenido un peso relevante otros servicios diferentes al meramente hospitalario (15).

Era esperable que los datos de todas las categorías fueran diferentes y superiores a los datos sobre salud. Ahora bien, la buena correlación existente entre ambos VBR indica que en el primer dato tiene un importante peso los servicios relacionados con la salud.

La evolución temporal del VBR presentó, en ambos casos, una gráfica en "v" con el punto de cambio prácticamente en la mediana de la distribución de la edad de la búsqueda, y que separaba 
las dos épocas en las que se segmentó el análisis estadístico. La primera época con tendencia decreciente y en la segunda con un claro crecimiento.

El interés inicial puede coincidir con las noticias de la implantación, en España, de las primeras unidades de hospitalización domiciliaria, que presenta un desarrollo irregular, pero que aparece como una de las prestaciones reconocidas en la cartera de servicios comunes del Sistema Nacional de Salud en 2006 (14). Lo que quedó patente es que, hoy en día, los servicios de atención domiciliaria (en cualquiera de sus vertientes) vuelve a ser un tema de alto interés y seguramente deben existir multitud de motivos que soporten este interés (mayor conocimiento de las unidades de hospitalización a domicilio, envejecimiento de la población española, etc.).

La evolución temporal presentó una gráfica en diente de sierra (según estacionalidad), ya observada en un estudio anterior (10), en la que no se pudo observar ningún hito de especial interés que respondiera a campañas de información puntuales que generalmente se traducen en un mayor interés de la población por el tema y, en consecuencia, un mayor número de búsquedas de información (16).

Posibles limitaciones: de acuerdo con Johnson \& Mehta (12), no contar con datos de uso reales e intervalos de tiempo más precisos disminuye la capacidad de pronóstico. Además, se echa en falta una mayor transparencia, ya que no existe información sobre los métodos específicos que Google utiliza para pronosticar estas tendencias. Aparte, como sugieren Cervellin et al. (17), los resultados que se obtienen en «Google Trends» pueden estar influenciados por el interés de los medios.

Por todo lo observado, se pudo concluir que los resultados obtenidos pueden representar una nueva fuente para conocer el interés de la población en la información sobre salud $(10,18)$. La evolución del VBR que se obtuvo probablemente se deba a un cambio de preocupación social y esté ligado al vaivén de la información sobre los servicios de asistencia sanitaria domiciliaria en España. Futuras investigaciones deberían corroborar estas conclusiones ya que no dejan de ser una hipótesis que el actual estudio no está en condiciones de despejar. Además, estos nuevos estudios pueden confirmar, o no, la utilidad y limitaciones de Google Trends en el seguimiento de la necesidad de información sobre salud de la población.

\section{BIBLIOGRAFÍA}

1. Sanz-Lorente M, Castejón Bolea R. Redes sociales: Recursos interactivos y la información sobre salud. Hosp Domic. 2019;3(4):269. DOI: 10.22585/hospdomic.v3i4.84

2. Sanz-Lorente M, Sanz-Valero J, Wanden-Berghe C. Tendencias temporales de los patrones de búsqueda de información sobre VIH/sida en España. Rev Esp Comun Salud. 2019;(Supl 2):S5260. DOI: $10.20318 /$ recs.2019.4554

3. Sanz-Lorente M, Sanz-Valero J, Wanden-Berghe C. La búsqueda de información y su asociación con la producción científica: el ejemplo de la sífilis y la gonorrea. Rev Cuba Inf Cienc Salud. 2019;30(4):e1401. DOI: 10.36512/rcics.v30i4.1401.g859

4. Eysenbach $\mathrm{G}$. Infodemiology and infoveillance: framework for an emerging set of public health informatics methods to analyze search, communication and publication behavior on the Internet. J Med Internet Res. 2009;11(1):e11. DOI: 10.2196/jmir.1157; PMID: 19329408

5. Mavragani A, Ochoa G, Tsagarakis KP. Assessing the methods, tools, and statistical approaches in Google Trends research: systematic review. J Med Internet Res. 2018;20(11):e270. DOI: 10.2196/jmir.9366; PMID: 30401664 
6. Pelat C, Turbelin C, Bar-Hen A, Flahault A, Valleron A-J. More diseases tracked by using Google Trends. Emerging Infect Dis. 2009;15(8):1327-8. DOI: 10.3201/eid1508.090299; PMID: 19751610

7. Johnson AK, Mehta SD. A comparison of Internet search trends and sexually transmitted infection rates using Google trends. Sex Transm Dis. 2014;41(1):61-3. DOI: 10.1097/ OLQ.0000000000000065; PMID: 24326584

8. Orellano PW, Reynoso JI, Antman J, Argibay O. Using Google Trends to estimate the incidence of influenza-like illness in Argentina. Cad Saude Publica. 2015;31(4):691-700. PMID: 25945979

9. Chiu APY, Lin Q, He D. News trends and web search query of HIV/AIDS in Hong Kong. PLoS One. 2017;12(9):e0185004. DOI: 10.1371/journal.pone.0185004; PMID: 28922376

10. Sanz-Lorente M, Wanden-Berghe C. Tendencias temporales de los patrones de búsqueda de información sobre cuidado domiciliario "Home Care" u hospitalario "Hospital Care" a través de Google. Hosp Domic. 2018;2(3):93-9. DOI: 10.22585/hospdomic.v2i3.47

11. Nuti SV, Wayda B, Ranasinghe I, Wang S, Dreyer RP, Chen SI, et al. The use of google trends in health care research: a systematic review. PLoS ONE. 2014;9(10):e109583. DOI: 10.1371/ journal.pone.0109583; PMID: 25337815

12. Johnson AK, Mehta SD. A comparison of Internet search trends and sexually transmitted infection rates using Google trends. Sex Transm Dis. 2014;41(1):61-3. DOI: 10.1097/ OLQ.0000000000000065; PMID: 24326584

13. Arias J, Muñoz L, Mias M, Estrada MD, Espallargues M. Avaluació de l'hospitalització a domicili: anàlisi de la situació a Catalunya en el període 2010-2014. Ann Med. 2016;99(4):169-73.

14. Estrada Cuxart O, Massa Domínguez B, Ponce González MÁ, Mirón Rubio M, Torres Corts A, Mujal Martínez A, et al. Proyecto HAD 2020: una propuesta para consolidar la hospitalización a domicilio en España. Hosp Domic. 2017;1(2):93-117. DOI: 10.22585/hospdomic.v1i2.13

15. Landers S, Madigan E, Leff B, Rosati RJ, McCann BA, Hornbake R, et al. The future of Home Health Care: a strategic framework for optimizing value. Home Health Care Manag Pract. noviembre de 2016;28(4):262-78. DOI: 10.1177/1084822316666368; PMID: 27746670

16. Ortiz-Martinez Y, Rios-González CM. Global impact of the World Hepatitis Day 2016: An evaluation using Google Trends. J Infect Public Health. 2017;10(5):690-1. DOI: 10.1136/sextrans-2017-053197; PMID: 28408645

17. Cervellin G, Comelli I, Lippi G. Is Google Trends a reliable tool for digital epidemiology? Insights from different clinical settings. J Epidemiol Glob Health. 2017;7(3):185-9. DOI: 10.1016/j. jegh.2017.06.001; PMID: 28756828

18. Tkachenko N, Chotvijit S, Gupta N, Bradley E, Gilks C, Guo W, et al. Google Trends can improve surveillance of Type 2 diabetes. Sci Rep. 2017;7(1):4993. DOI: 10.1038/s41598-017-05091-9; PMID: 28694479 